JRRD

\title{
Can static interface pressure mapping be used to rank pressure- redistributing cushions for active wheelchair users?
}

\author{
James Hollington, MSc; ' Susan J. Hillman, MSc \\ Southeast Mobility and Rehabilitation Technology (SMART) Centre, Edinburgh, Scotland
}

\begin{abstract}
Interface pressure mapping (IPM) is a clinical tool that assists the selection of seat cushions for pressure management for wheelchair users. Clinical pressure measurements are almost always made under static sitting conditions, although this does not consider the time-dependent properties of some cushion materials that may behave differently under the dynamic conditions of self-propulsion. This study investigated the potential for such differences by collecting seat IPM measurements from eight wheelchair users using four different seat cushion designs during static sitting and self-propulsion. Mean pressure corresponding to the approximate anatomical location of the ischial tuberosities was used to rank the four cushions under the two conditions. The two sets of rankings for each participant were then compared using correlation. Dynamic data from four participants was judged too inconsistent to be interpreted reliably and demonstrates the practical difficulties associated with dynamic IPM measurement when variations in individual propulsion technique cannot be controlled. Strong correlations were observed between rank orders for the remaining four participants and suggest that the statically derived pressure measures can be used for clinical decision making when selecting cushions for self-propelling wheelchair users.
\end{abstract}

Key words: cushion, dynamic, foam, interface, measurement, pressure, propulsion, seating, static, wheelchair.

\section{INTRODUCTION}

"Pressure ulcers are a major secondary medical complication experienced by many people with physical impairments” [1]. Pressure ulcers are a huge problem within health services, both in terms of quality of life for the patient with the pressure ulcer and in financial costs to the health service providing care. Studies show the prevalence of sitting-acquired pressure ulcers in wheelchair users ranges between 23.0 and 17.9 percent [2-3]. Therefore, it is imperative to select a correct pressureredistributing surface for people who are at risk of developing pressure ulcers. "Inappropriate selection not only wastes capital resources, but it can also be detrimental to the patient" [4].

There are a vast number of commercial wheelchair cushions on the market utilizing different materials and constructions. Cushion construction plays an important role in their pressure-redistributing qualities. The ability of a cushion to provide pressure redistribution is governed by three factors: materials used, construction or design, and fit of the cushion to the person. Five main materials used in wheelchair cushion construction are standard foam, viscoelastic foam, gel, viscous fluid, and air. The

Abbreviations: ANOVA = analysis of variance, CV = coefficient of variation, IPM = interface pressure mapping, IT = ischial tuberosity, PRI = Pressure Relief Index, PTI = Pressure Time Integral.

*Address all correspondence to James Hollington, MSc; Southeast Mobility and Rehabilitation Technology (SMART) Centre, Astley Ainslie Hospital, 133 Grange Loan, Edinburgh, EH9 2HL, United Kingdom; +44-(0)131-537-9441; fax: +44-(0)131-537-9552.

Email: james.hollington@nhslothian.scot.nhs.uk http://dx.doi.org/10.1682/JRRD.2011.10.0192 
pressure-redistributing properties of some of these materials are temperature and/or time dependent. This means that some materials may need time to "settle" and/or may require warming to a certain temperature for their optimal pressure-redistributing properties to come into effect. Therefore, it is logical to expect that the pressure-redistributing properties for some construction materials and designs may be different under dynamic (cyclic) loading than under static conditions.

Interface pressure mapping (IPM) is a readily available technology to assist clinicians in pressure care assessment. In order to help seating practitioners assess the seated posture and pressure distribution needs of wheelchair users, a variety of real-time objective IPM devices are available [1]. "Pressure mapping systems can add a powerful tool to a therapist's seating and positioning evaluations," and by using pressure mapping systems the clinician can "readily visualize how uniformly pressure is distributed over the seating surface" [5]. IPM data can be used to rank support surfaces, indicating their relative merits in terms of effectiveness of the cushions to provide the required pressure-redistribution characteristics for an individual user.

In the evaluation of these pressure maps, however, it is important to focus on the most clinically relevant aspects of the map. McLeod expresses the need for pressure map protocols to test the following key pressure areas for cushion evaluation: ischial tuberosities (ITs), greater trochanters, and sacrum [6]. Research has illustrated that pressure ulcers commonly develop at the ITs [3]. In contrast, under dynamic conditions Tam et al. studied the movement of the pelvis and the redistribution of interface pressure during manual wheelchair propulsion [7]. They found that for both the group with spinal cord injury and the nondisabled group, "the peak pressure locations did not concur exactly with the ischial tuberosities (19.2 $\pm 11.7 \mathrm{~mm}$ behind the peak pressure locations)." However, even though they measured sagittal plane pelvic rocking, their data imply little movement of the areas of peak pressure during propulsion. Mayall and Desharnais stated, "Sitting is a dynamic, not a static behavior. When seated, many different postures are assumed and vary according to the activity performed" [8].

An additional consideration when pressure mapping for active users is the mechanical response of the cushion materials. If, as discussed earlier, a settling effect is required for some cushions in order for them to provide optimum pressure redistribution, they may not be the most appropriate for a dynamic activity such as propelling a wheelchair. Therefore, although "the duration of pressure is a significant variable in the development of pressure ulcers” [9], some pressure-redistributing support surfaces may give poor pressure redistribution if not allowed to settle. Despite this, however, it is normal clinical practice for seat interface pressure distributions to be measured with the patient in a fixed (static) position [10-11].

Dynamic pressure-redistribution characteristics have featured in some previous studies $[4,10,12-14]$, but the inclusion of time-dependent information necessitates a more complex approach to measurement. Commonly, measures have been reported in terms of the pressuretime cycle. Two frequently used performance indices for dynamic pressure-redistribution characteristics are the Pressure Relief Index (PRI) and the Pressure Time Integral (PTI) (also known as Pressure Impulse). Both the PRI and PTI do not give a measure for peak pressures but instead measure overall cycle pressure relief/redistribution. The PRI reports the percentage of the cycle during which the surface gives relief, where relief is defined arbitrarily (commonly 40, 30, or $20 \mathrm{mmHg}$ ). Historically, this measure is used for analyzing dynamic or alternating pressure cushions with low cycle frequencies. The PTI, by contrast, is the area under the pressure-time curve and has been used to measure dynamic pressure at higher frequencies. This parameter represents accumulated pressure and is a measure of the total applied pressure per standard time period. Therefore, under conditions of steady-state propulsion, the PTI is effectively equivalent to mean pressure.

Among studies considering dynamic conditions, Kernozek and Lewin found that peak pressure during static sitting was statistically lower than the maximum peak pressure during a dynamic cycle [10]. However, the same study showed that peak pressure varied throughout the dynamic cycle to a level below that of static loading conditions. The study used 15 subjects but made test comparisons on only one cushion. Kernozek and Lewin also investigated three different cushion compositions (foam, gel, and air) during propulsion and found that "cushion performance may enhance the effectiveness of seating systems in minimising pressure fluctuations" [11]. No comparisons were made with static loading, however. Such results hint that we may not be able to infer the dynamic performance of a cushion from statically derived pressure measures. 
To date, however, no studies have investigated whether the optimal pressure-redistributing cushion identified by ranking static IPM measurements reflects the optimal surface for dynamic sitting. Therefore, this study aims to investigate whether static IPM is reliable for ranking pressure-redistributing cushions for active (dynamic) wheelchair users by comparing the ranking of pressure-redistributing cushions ranked by static IPM with the ranks obtained through dynamic IPM.

\section{METHODS}

We recruited active self-propelling wheelchair users from a wheelchair basketball training squad, excluding users with a history of pressure ulcers during the 6 months prior to the study. We took IPM measures from each participant on four different wheelchair seat cushions under static and dynamic propulsion conditions. The four cushions were constructed as follows: (1) CM40H polyurethane foam (polyfoam), (2) Sunmate viscoelastic foam (Dynamic Systems Inc; Leicester, North Carolina), (3) polyfoam with recess under ITs containing a Flo-Tech Plus silicone gel pack (Invacare; Elyria, Ohio), and (4) polyfoam with recess under ITs containing a ROHO dry flotation insert from the Nexus Spirit cushion (The ROHO Group; Belleville, Illinois). All cushions used were $75 \mathrm{~mm}$ high and not upholstered. IPM measures were made using a Force Sensitive Applications (Vista Medical; Winnipeg, Canada) IPM system with 256 piezo-resistive force sensors sampling at $10 \mathrm{~Hz}$. We collected all data with the user and wheelchair positioned on a wheelchair ergometer. We visually compared interface pressure oscillations and found them to be similar under dynamic conditions when propelling on the ergometer and on a smooth surface.

We collected data from each cushion according to the following protocol:

1. Each participant first sat on the $75 \mathrm{~mm}$ polyfoam cushion and propelled the wheelchair at a fast speed that he or she could maintain at a steady state for $30 \mathrm{~s}$. We noted the speed from a speedometer connected to the ergometer.

2. The participant sat still for 2 min on the first cushion selected for testing while we collected data.

3. The participant propelled the wheelchair at the same speed as established in phase 1 of testing using the speedometer feedback to adjust speed accordingly. We required participants to maintain constant speed for at least 15 propulsion cycles from rim strike to rim strike.

4. We encouraged participants to relieve pressure by leaning forward.

5. Phases 2 to 4 were repeated for the remaining cushions in random order.

This process was then repeated twice more to yield three sets of static and dynamic data for each cushion for each participant. Each cushion was made to measure and set up/adjusted in accordance with normal clinical practice prior to data collection.

We examined graphical displays of the static and dynamic pressure distributions for each trial for consistency between trials for each participant, cushion, and static/dynamic combination. We excluded participants showing marked inconsistency for one or more sets of trials from further analysis. We then computed mean pressure of the two peak areas (corresponding to the approximate anatomical location of the ITs) for each data sample. We calculated each peak pressure area as the mean for four sensors with each sensor covering approximately 1 in. $^{2}$ (625 $\mathrm{mm}^{2}$ ). We then computed static measures for each trial from mean pressure during a $10 \mathrm{~s}$ period (100 samples) of steady-state sitting. We calculated the mean of the three repeated trials for each cushion/participant combination. We computed dynamic data as the mean of between 50 and 100 samples ( 5 and $10 \mathrm{~s}$ ) of repeatable dynamic data as judged by visual inspection of a plot of the data points against time and summarized these as the mean of the three trials. Visual inspection of the IPM system's graphical display showed that during propulsion the locations of the peak pressures did not move but the magnitude of pressure fluctuated. These areas were also located within the area of the same four sensors as were identified during static sitting. Therefore, we used the same areas to calculate mean peak pressure areas during static conditions during propulsion (dynamic conditions), focusing on the areas known to be at risk from pressure ulcer development [3,6-7]. We decided to use the mean value rather than a measure more traditionally associated with dynamic IPM measures, such as the PTI, because the IPM waveform under dynamic propulsion conditions is a complex waveform containing undetermined high frequencies and, by Nyquist's theorem, could not be accurately reproduced for analysis because of limitations in the sampling rate available with the IPM system. 
We computed coefficient of variation (CV) from the three sample means for each participant/cushion/condition combination to check repeatability of data. We excluded data sets showing any $\mathrm{CV}$ of $>15$ percent from analysis. We derived this criterion from McLeod [6], who suggested that a CV of $>10$ percent indicates poor repeatability in IPM measurements made with a static mannequin. We relaxed McLeod's criterion of 10 percent to 15 percent in this study to account for the additional variability expected with human participants, especially under dynamic conditions.

We ranked cushions by mean pressure under the ITs for each participant under static and dynamic conditions. We then computed one-tailed Spearman rank correlation coefficients between static and dynamic pressure for each participant. The static and dynamic data sets for each participant were also separately tested using one-way analysis of variance (ANOVA) with post hoc protected $t$-tests to disclose the potential of incorrect ranking of cushions.

\section{RESULTS}

We initially recruited eight participants to the study (Table 1). However, the data from participant 2 showed very marked inconsistency between trials of the same condition and were excluded from further analysis. Furthermore, the CVs of some of the sample means for each participant/cushion/condition combination were $>15$ percent for participants 1,3 , and 4 , so we also excluded the data from these participants.

For the remaining data (Table 2), the Spearman rank correlation coefficients between static and dynamic pressure

Table 1.

Details of participants.

\begin{tabular}{|c|c|c|c|c|}
\hline Participant & $\begin{array}{l}\text { Age } \\
(\mathrm{yr})\end{array}$ & Sex & Diagnosis & $\begin{array}{c}\text { Wheelchair } \\
\text { Usage }\end{array}$ \\
\hline 1 & 28 & $\mathrm{M}$ & SCI (L10) & Full-time \\
\hline 2 & 17 & M & Spina bifida & Full-time \\
\hline 3 & 35 & M & $\begin{array}{l}\text { Hip disarticulation } \\
\text { (right) }\end{array}$ & Full-time \\
\hline 4 & 27 & M & Arthrogryposis & Full-time \\
\hline 5 & 48 & $\mathrm{~F}$ & $\begin{array}{l}\text { Bilateral transtibial } \\
\text { amputation }\end{array}$ & Part-time \\
\hline 6 & 29 & $\mathrm{~F}$ & Spina bifida & Full-time \\
\hline 7 & 37 & M & Spina bifida & Full-time \\
\hline 8 & 26 & M & None & 3-6 h/wk \\
\hline
\end{tabular}

measures were 1 for participants 5,6 , and 7 , denoting perfect correlation (Table 3). The $p$-values for these correlations were 0.04 , denoting that they were individually significant at the $p<0.05$ level. For participant 8 , the Spearman rank correlation coefficient was 0.8 with a $p$-value of 0.17 . This corresponded to a transposition in the ranking of two of the cushions. Note that ranks were more poorly correlated when compared between subjects.

The ANOVA tests and protected $t$-tests within static and dynamic data sets for each participant showed nonsignificant differences between some cushions at the $p<$ 0.05 level in all groups; hence, there was poor confidence in the rank orders as determined by the means. However, this may also be explained by the power of the post hoc $t$ tests, which was as low as 5 percent for some tests because of large variability between trials for some cushions, giving a high probability of a type 2 error.

\section{DISCUSSION}

These results suggest that static and dynamic IPM measures result in similar rankings for wheelchair seating cushions. If this conclusion can be extended across the full range of cushions and users, it is of great clinical usefulness because it means that the prescribing clinician can use static IPM measures to make informed decisions about the potential pressure exposure of active users, which are much more clinically practicable than dynamic measures. It also obviates the need to consider the complexities of the dynamic responses of wheelchair seating materials. Such a conclusion cannot be asserted confidently, however, because this study has also demonstrated considerable uncertainties inherent in the process of making IPM measurements. This is evidenced by the unreliability of the data from four of the participants and also the difficulty in confidently ranking the cushions for the remaining participants given the large variability in the repeated pressure measures.

Note that there are a variety of reasons for the unreliability of the data collected in the study. We discarded the data from participant 2, for example, because of large inconsistencies as judged by visual inspection of the pressure maps for the same cushion under static conditions. This inconsistency was seen as symmetrical loading across the seat surface on some occasions, but very asymmetrical loading on other occasions, with negligible pressure readings being observed under the IT and greater 
Table 2.

Pressure data used to generate cushion rankings for four participants under static and dynamic conditions, where $1=$ lowest pressure and $4=$ highest pressure.

\begin{tabular}{|c|c|c|c|c|c|c|}
\hline \multirow{2}{*}{ Participant } & \multirow{2}{*}{$\begin{array}{c}\text { Condition } \\
\text { (mmHG) }\end{array}$} & \multirow{2}{*}{ Repeat } & \multicolumn{4}{|c|}{ Cushion } \\
\hline & & & Polyfoam & Viscoelastic & Gel Insert & Air Insert \\
\hline \multirow[t]{9}{*}{5} & Static & $\mathrm{I}$ & 61 & 80 & 75 & 75 \\
\hline & & II & 62 & 81 & 62 & 75 \\
\hline & & Mean \pm SD & $63.10 \pm 2.53$ & $77.73 \pm 3.99$ & $67.97 \pm 6.81$ & $72.93 \pm 3.72$ \\
\hline & & CV (\%) & 4.01 & 5.13 & 10.02 & 5.11 \\
\hline & & II & 52 & 72 & 53 & 59 \\
\hline & & III & 57 & 65 & 58 & 59 \\
\hline & & Mean \pm SD & $54.13 \pm 2.50$ & $69.52 \pm 3.71$ & $58.59 \pm 6.19$ & $60.14 \pm 1.47$ \\
\hline & & CV (\%) & 4.61 & 5.33 & 10.57 & 2.45 \\
\hline & & Rank & 1 & 4 & 2 & 3 \\
\hline & & CV (\%) & 4.19 & 4.75 & 11.40 & 0.88 \\
\hline & & Rank & 3 & 4 & 1 & 2 \\
\hline & Dynamic & $\mathrm{I}$ & 46 & 52 & 37 & 41 \\
\hline & & II & 43 & 51 & 38 & 38 \\
\hline & & III & 43 & 55 & 38 & 36 \\
\hline & & Mean \pm SD & $44.04 \pm 1.30$ & $52.40 \pm 2.04$ & $37.77 \pm 1.08$ & $38.12 \pm 2.66$ \\
\hline & & CV (\%) & 2.96 & 3.89 & 2.86 & 6.98 \\
\hline & & Rank & 3 & 4 & 1 & 2 \\
\hline \multirow[t]{5}{*}{7} & Static & I & 95 & 102 & 70 & 63 \\
\hline & & II & 88 & 80 & 62 & 72 \\
\hline & & Mean \pm SD & $93.02 \pm 3.15$ & $83.04 \pm 6.99$ & $69.72 \pm 7.52$ & $65.99 \pm 2.90$ \\
\hline & & CV (\%) & 3.38 & 8.42 & 10.79 & 4.40 \\
\hline & & Rank & 4 & 3 & 2 & 1 \\
\hline \multirow[t]{12}{*}{8} & Static & I & 72 & 75 & 63 & 54 \\
\hline & & II & 72 & 74 & 55 & 46 \\
\hline & & III & 73 & 78 & 61 & 62 \\
\hline & & Mean \pm SD & $72.40 \pm 0.70$ & $75.47 \pm 2.14$ & $59.57 \pm 3.99$ & $54.17 \pm 7.74$ \\
\hline & & CV (\%) & 0.97 & 2.84 & 6.71 & 14.30 \\
\hline & & Rank & 3 & 4 & 2 & 1 \\
\hline & Dynamic & $\mathrm{I}$ & 65 & 61 & 51 & 44 \\
\hline & & II & 65 & 61 & 43 & 42 \\
\hline & & III & 63 & 62 & 45 & 46 \\
\hline & & Mean \pm SD & $64.33 \pm 1.48$ & $61.25 \pm 0.38$ & $46.36 \pm 4.07$ & $44.01 \pm 2.09$ \\
\hline & & CV (\%) & 2.30 & 0.62 & 8.79 & 4.75 \\
\hline & & Rank & 4 & 3 & 2 & 1 \\
\hline
\end{tabular}


JRRD, Volume 50, Number 1, 2013

Table 3.

Rankings of cushions by mean interface pressure under ischial tuberocities for four participants under static and dynamic conditions, where $1=$ lowest pressure and 4 = highest pressure.

\begin{tabular}{|c|c|c|c|c|c|c|c|c|}
\hline \multirow{2}{*}{ Cushion } & \multicolumn{2}{|c|}{ Participant 5} & \multicolumn{2}{|c|}{ Participant 6} & \multicolumn{2}{|c|}{ Participant 7} & \multicolumn{2}{|c|}{ Participant 8} \\
\hline & Static & Dynamic & Static & Dynamic & Static & Dynamic & Static & Dynamic \\
\hline Polyfoam & 1 & 1 & 3 & 3 & 4 & 4 & 3 & 4 \\
\hline Viscoelastic & 4 & 4 & 4 & 4 & 3 & 3 & 4 & 3 \\
\hline Air Insert & 3 & 3 & 2 & 2 & 1 & 1 & 1 & 1 \\
\hline
\end{tabular}

trochanter of one side. Such pressure maps suggest that this participant is not consistent when transferring into his wheelchair and does not adjust his posture after transferring to optimize distribution of pressure. Such inconsistency has created a confounding factor for these data, but more detailed study of this effect may provide new insights into the risk factors for pressure ulcers, not only for active users but also for those with more extensive impairments whose seated posture depends on the skill of others in effecting hoisted transfers.

We also discarded the data for participants 1 , 3, and 4 for reasons of inconsistency. For these individuals, the data appeared consistent from examination of static pressure maps but failed to achieve a $\mathrm{CV}$ for the sample means of $<15$ percent. As would be expected, these large $\mathrm{CV}$ values were almost exclusively observed under dynamic conditions, with only one static condition CV (participant 3) of $>15$ percent. However, these data also hinted that inconsistency in dynamic pressure readings may be an individual propulsion trait to some extent, with the dynamic CVs of one participant (participant 4) ranging from 29.0 to 78.1 percent, showing markedly greater variability than the data from participant 1 , which ranged from 13.3 to 30.6 percent, and participant 3 , which ranged from 15.9 to 28.8 percent. This suggestion is supported by observations made during the study that some participants were not able to maintain an invariant propulsion technique. This highlights the potential sensitivity of dynamic IPM measures to variations in technique, which may include changes in trunk forward lean, differing rim strike positions, variation in push force, and push frequency.

For the remaining data from participants 5 through 8, the results of the ANOVA and protected $t$-tests meant that the differences between cushions were insignificant at the $p<0.05$ level, hence the rank orders could not be asserted with confidence. This was because the variability of the repeated measures was large compared with the differences between the means. However, the standard deviations and differences in means found in this study would require the number of repeated measures under each condition to have been at least an order of magnitude greater for a statistical power of 80 percent. This clearly would have presented its own difficulties with the potential for fatigue in a study demanding such a high level of physical exertion.

Given these large uncertainties, however, the correlation of static and dynamic cushions rankings for individuals is perhaps surprisingly good, with perfect correlations being noted for participants 5, 6, and 7 and the transposition of only two adjacently ranked cushions for participant 8 . The interpretation of this finding is difficult given the uncertainties but may suggest that when an individual's variability can be limited to beneath an appropriate threshold value, the ranking of cushions using IPM measurements can be reliable. If this is true, it also suggests that ranking by dynamic IPM measures gives similar results to ranking by static IPM measures. However, correlation of rankings was poorer between different participants (Table 3), with no correlations found to be perfect. This may reflect the different diagnoses of these individuals and, as such, would be consistent with the findings of Swain [15], who found different cushion rankings under static conditions for the group of participants with flaccid spinal cord injury than for the other groups in that study. Therefore, these results reiterate the important finding that cushions must be selected according to the particular characteristics of the intended user and that too general an approach to cushion selection for pressure management is unlikely to lead to optimum prescription for all.

A further important consideration in this study is the choice of reporting method for the dynamic data. The interface pressure under these conditions is periodic, and the Figure illustrates a typical cycle of this complex waveform, taking rim strike as the cycle start and end points. A summary measure of this pressure is therefore required, and a variety of measures have been considered 


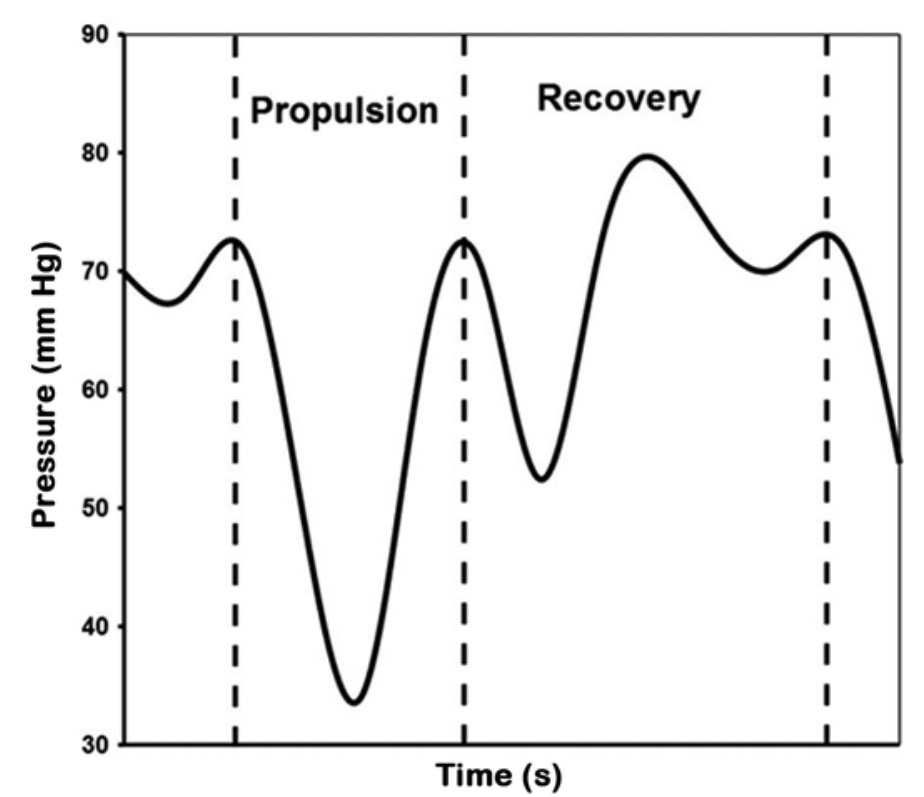

Figure.

Mean interface pressure under ischial tuberosities against time during wheelchair propulsion for participant 8. One cycle indicated by dotted lines.

in previous studies. These include maximum, minimum, and mean pressure with time [16]; PRI, which is the proportion of the cycle for which the pressure is above a defined threshold [4,17]; and PTI. Unfortunately, no single measure can provide a complete picture of the interacting risk factors of peak pressure, duration of loading, and recovery periods, and the challenge of interpreting these measures clinically is exacerbated by apparently contradictory theories such as that of Rithalia [16], who proposes that cyclic loading prevents necrosis by allowing oxygen and nutrients to reach the cells, and Bader [18], who cites reperfusion damage as a pressure sore risk factor. For this study, therefore, we used the expedient of average since this provided the most general summary of the pressure exposure without the need for a high data sampling rate. We acknowledge, however, that other measures may have given different results, and further work should include the identification of a clinically meaningful summary of dynamic pressure.

\section{CONCLUSIONS}

In conclusion, this study has found that different cushioning materials rank similarly irrespective of whether mean static or mean dynamic pressure is used for the ranking. In addition, a large amount of variability has been found, which highlights the practical difficulties in measuring dynamic interface pressures. Therefore, if dynamic pressure measures are to be used, new approaches to collecting these data are required to ensure information is reliable and meaningful.

\section{ACKNOWLEDGMENTS}

\section{Author Contributions:}

Study concept and design: J. Hollington.

Acquisition of data: J. Hollington.

Analysis and interpretation of data: J. Hollington, S. J. Hillman.

Drafting of manuscript: J. Hollington, S. J. Hillman.

Critical revision of manuscript for important intellectual content: J. Hollington, S. J. Hillman.

Statistical analysis: J. Hollington, S. J. Hillman.

Financial Disclosures: The authors have declared that no competing interests exist.

Institutional Review: The National Disability Authority (NDA) does not have in place an ethics approval process for research being funded by bodies outside of the NDA.

Funding/Support: This study was conducted using technical and equipment support from Enable Ireland SeatTech, Dublin, Ireland.

Participant Follow-up: The authors have no plans to notify the study subjects of this publication of this article because of a lack of contact information.

\section{REFERENCES}

1. Nicholson G, Bain D, Ferguson-Pell M. Practical experience in using draft ISO (CD 16840-2) Test methods for wheelchair seating-part 2: Test methods for devices intended to manage tissue in practice. European Pressure Ulcer Advisory Panel. 2003;5:97-98.

2. Raghavan P, Raza WA, Ahmed YS, Chamberlain MA. Prevalence of pressure sores in a community sample of spinal injury patients. Clin Rehabil. 2003;17(8):879-84.

[PMID:14682560] http://dx.doi.org/10.1191/0269215503cr692oa

3. Sumiya T, Kawamura K, Tokuhiro A, Takechi H, Ogata H. A survey of wheelchair use by paraplegic individuals in Japan. Part 2: Prevalence of pressure sores. Spinal Cord. 1997;35(9):595-98. [PMID:9300965] http://dx.doi.org/10.1038/sj.sc.3100467

4. Rithalia S, Kenney L. World Wide Wounds: The art and science of evaluating patient support surfaces [Internet]. Salford (England): University of Salford School of Health Care Professions; 2001 [updated 2001 Sep; cited 2011 Jul 7]. Available 
from: http://www.worldwidewounds.com/2001/september/ Rithalia-and-Kenney/Evaluating-Support-Surfaces.html

5. Ferguson-Pell M, Cardi M. Pressure mapping systems. TeamRehab Report. 1992;3:28-32.

6. McLeod A. Draft guidelines for the laboratory evaluation of pressure-redistributing support surfaces. EPUAP Review. 2002;4:8-12.

7. Tam EW, Mak AF, Lam WN, Evans JH, Chow YY. Pelvic movement and interface pressure distribution during manual wheelchair propulsion. Arch Phys Med Rehabil. 2003; 84(10):1466-72. [PMID:14586913]

http://dx.doi.org/10.1016/S0003-9993(03)00269-7

8. Mayall JK, Desharnais G. Positioning in a wheelchair: A guide for professional caregivers of the disabled adult. 2nd ed. Thorofare (NJ): SLACK; 1995.

9. Cook AM, Hussey SM. Principles of seating for pressure management. In: Assistive technologies: Principles and practice. 2nd ed. St. Louis (MO): Mosby; 2002. p. 189-204.

10. Kernozek TW, Lewin JE. Seat interface pressures of individuals with paraplegia: influence of dynamic wheelchair locomotion compared with static seated measurements. Arch Phys Med Rehabil. 1998;79(3):313-16. [PMID:9523784]

11. Kernozek TW, Lewin JE. Dynamic seating interface pressures during wheelchair locomotion: influence of cushion type. Occup Ther J Res. 1998;18:182-92.

12. Beil TL, Street GM, Covey SJ. Interface pressures during ambulation using suction and vacuum-assisted prosthetic sockets. J Rehabil Res Dev. 2002;39(6):693-700. [PMID:17943671]

13. Clark M, Rowland LB. Comparison of contact pressures measured at the sacrum of young and elderly subjects. J Biomed Eng. 1989;11(3):197-99. [PMID:2724940] http://dx.doi.org/10.1016/0141-5425(89)90140-4
14. Rithalia SV. Pressure sores: methods used for the assessment of patient support surfaces. Clin Rehabil. 1991;5: 323-29. http://dx.doi.org/10.1177/026921559100500409

15. Swain ID. Wheelchair cushions: Static and dynamic: A comparative evaluation special issue. London (England): Medical Devices Agency; 1997.

16. Rithalia SV. Assessment of pressure relief characteristics in alternating pressure air cushions. Int J Rehabil Res. 1997; 20(2):205-8. [PMID:9226505] http://dx.doi.org/10.1097/00004356-199706000-00011

17. Rithalia S. Know how-alternating pressure relief. Nurs Times. 1997;93(46):76-77. [PMID:9418495]

18. Bader DL. The recovery characteristics of soft tissues following repeated loading. J Rehabil Res Dev. 1990;27(2): 141-50. [PMID:2366198] http://dx.doi.org/10.1682/JRRD.1990.04.0141

Submitted for publication October 17, 2011. Accepted in revised form May 17, 2012.

This article and any supplementary material should be cited as follows:

Hollington J, Hillman SJ. Can static interface pressure mapping be used to rank pressure-redistributing cushions for active wheelchair users? J Rehabil Res Dev. 2013; 50(1):53-60.

http://dx.doi.org/10.1682/JRRD.2011.10.0192

ResearcherID: James Hollington, MSc: F-7000-2011; Susan J. Hillman, MSc: F-4105-2012

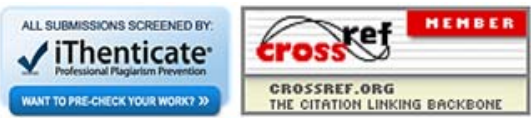

